\title{
Micellar dye shuttle between water and an ionic liquid $\dagger$
}

\author{
Carlos Guerrero-Sanchez, $\dagger^{* a b}$ Daan Wouters, ${ }^{a b}$ Stephanie Hoeppener, ${ }^{a c}$ Richard Hoogenboom $\oint^{a b}$ \\ and Ulrich S. Schubert $* a b c$
}

\author{
Received 21st November 2010, Accepted 4th January 2011 \\ DOI: 10.1039/c0sm01352h
}

The reversible transfer of poly(2-ethyl-2-oxazoline)-block-poly(2-nonyl-2-oxazoline) nanocarriers comprising encapsulated dyes is demonstrated between water and an ionic liquid. This dye transfer concept is shown to be applicable for loading and delivery of dye molecules as well as to provide a protective environment for the encapsulated dye.

\section{Introduction}

Recently the utilization of ionic liquids (ILs) as new solvents ${ }^{1-9}$ for the preparation of self-assembled nanostructures (micelles) formed by amphiphilic block copolymers has triggered attractive new possibilities for the design of advanced and "smart" polymer based physico-chemical systems. ${ }^{10-21}$ Interestingly, the thermoreversible transfer of block copolymer micelles and nanocarriers between a hydrophobic IL phase and an aqueous phase was recently demonstrated based on the decreased solubility of the hydrophilic block in aqueous solution with increasing temperature. $^{10-13}$

Block copolymer micelles and dendritic core-shell architectures have been proposed as encapsulating, phase-transfer, and delivery vehicles of molecules and nano-objects, ${ }^{22-30}$ including catalysts, ${ }^{26,27}$ drugs showing a steady release process, ${ }^{28}$ high loading capacity systems using star-shaped unimolecular micelles, ${ }^{23,27,28,30}$ and organic molecules in a $\mathrm{pH}$-responsive biphasic system. ${ }^{25}$ Most of these previous reports describe the delivery of encapsulated moieties in multi-phase or heterogeneous systems by an irreversible process often based on the destruction of the carrier.

The combination of block copolymer nanocarrier transfer between water and ILs with the encapsulation of guest molecules

${ }^{a}$ Laboratory of Macromolecular Chemistry and Nanoscience, Eindhoven University of Technology, P.O. Box 513, 5600 MB Eindhoven, The Netherlands

${ }^{b}$ Dutch Polymer Institute (DPI), John F. Kennedylaan 2, 5612 AB Eindhoven, The Netherlands

${ }^{c}$ Laboratory of Organic and Macromolecular Chemistry (IOMC) and Jena Center for Soft Matter (JCSM), Friedrich-Schiller-University Jena, Humboldtstrasse 10,07743 Jena, Germany.E-mail: ulrich.schubet@ uni-jena.de

$\dagger$ Electronic supplementary information (ESI) available: Additional discussion on the size of the nanocarriers. See DOI: $10.1039 / \mathrm{c} 0 \mathrm{sm} 01352 \mathrm{~h}$ \$ Current address: CSIRO, Materials Science and Engineering Division, Bag 10, Clayton South MDC, 3169 Victoria, Australia. E-mail: carlos. guerrero-sanchez@csiro.au

$\S$ Current address: Supramolecular Chemistry group, Department of Organic Chemistry, Ghent University, Krijgslaan 281 S4, B-9000 Ghent, Belgium is envisioned to lead to a reversible shuttling of the guest between the phases, which could be beneficial for transport or delivery and reloading of catalysts or precious reagents as well as an easy recovery of the nanocarrier shuttle or of permanently loaded nanocarrier catalysts. ${ }^{31}$

Here, we report dye encapsulation in poly(2-ethyl-2-oxazoline) $)_{67}-b$-(2-nonyl-2-oxazoline) $)_{33} \quad\left(\right.$ PEtOx $\left._{67}-b-\mathrm{NonOx}_{33}\right)$ (Scheme 1) block copolymer nanocarriers, which can be reversibly shuttled between water and 1-butyl-3-methylimidazolium hexafluorophosphate $\left(\mathrm{BMI}_{-} \mathrm{PF}_{6}\right.$, Scheme $)$ IL. ${ }^{11}$ Furthermore, the thermoreversible transfer and/or release of the dye guest molecules encapsulated into block copolymer nanocarriers are reported, whereby reversible shuttling, one-direction deliveries as well as reloading of the carriers are demonstrated. Recently, the reversible transfer of covalently dye-loaded block copolymer micelles was utilized to gain a better understanding of the micellar shuttling process, ${ }^{32,33}$ which is distinctly different from our current study focusing on guest transfer, delivery and reloading.

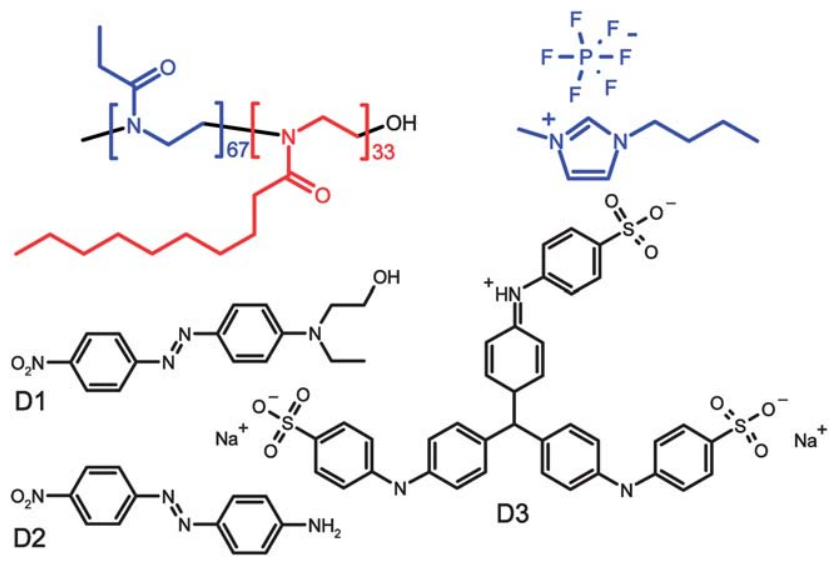

Scheme 1 Schematic representation of the $\mathrm{PEtOx}_{67}-b-\mathrm{PNonOx}_{33}$ block copolymer, the BMI-PF 6 IL and the disperse red 1 (D1), disperse orange (D2) and methyl blue (D3) dyes. 


\section{Experimental section}

\section{Materials}

1-Butyl-3-methylimidazolium hexafluorophosphate and 1-butyl3-methylimidazolium trifluoromethanesulfonate $\left(\mathrm{BMI}-\mathrm{CF}_{3} \mathrm{SO}_{3}\right)$ ILs were obtained from Solvent-Innovation $\mathrm{GmbH}$ as a kind gift. Both ILs were of synthesis grade and dried under vacuum at $40{ }^{\circ} \mathrm{C}$ for at least 3 days before use. The rest of the materials were utilized as received from different suppliers.

\section{Characterization techniques}

Dynamic light scattering (DLS) measurements were performed at $25^{\circ} \mathrm{C}$ at $90^{\circ}$ on a Malvern CGS-3 apparatus equipped with

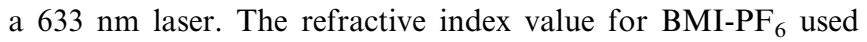
during the analysis was 1.41 (measured value) whereas the used value of viscosity for BMI-PF 6 was $352 \mathrm{mPa}$ s (value obtained from the supplier). The hydrodynamic radius $\left(R_{\mathrm{h}}\right)$ and the polydispersity index (PDI) of the nanocarriers were calculated from a cumulant analysis. The distribution of the hydrodynamic radii was obtained by a deconvolution of the data with the CONTIN algorithm.

Transmission electron microscopy (TEM) images were recorded on a Technai $G^{2}$ Sphera (FEI) electron microscope using an acceleration voltage of $200 \mathrm{kV}$. The IL nanocarrier solution was drop-cast on the TEM-grid followed by washing with water to remove excess of the IL.

Ultraviolet-visible (UV-Vis) spectroscopy was performed on a FlashScan 520 (Analytik Jena, Germany) in 96 well microtiter plates (poly(propylene), flat bottom) from Greiner (Greiner BioOne, Germany) in the range from 250 to $800 \mathrm{~nm}$. All spectra were referenced to an empty microtiter plate and utilized four flashes. For the investigation of the phase transfer process of the guest molecules encapsulated into the cores of the self-assembled polymeric nanocarriers, UV-Vis measurements of the two phases of the systems described in this contribution were recorded during the different stages of the experiments in order to monitor the presence and/or absence of the encapsulated molecules within the phases of the system. For this study, it was important to determine the UV-Vis absorption properties of the investigated dispersion of the nanocarriers before the addition of any dye moieties. In this regard, UV-Vis measurements demonstrated that neither the pure solvents $\left(\mathrm{BMI}-\mathrm{PF}_{6}\right.$ and water) nor the pure dispersion of nanocarriers show strong absorption bands in the range of 350 to $900 \mathrm{~nm}$, thus allowing the analysis with respect to the presence and/or absence of the encapsulated molecules within the phases of the system.

$\mathrm{pH}$ measurements of micellar dispersions as well as pure substances were performed in poly(propylene) containers using an HI 8417 Hanna Instruments bench-top pH meter supplied with an HI 1131B glass-body combination pH electrode.

Size-exclusion chromatography (SEC) measurements of the $\mathrm{PEtOx}_{67}-b$-PNonOx 33 diblock copolymer were performed on a Waters SEC system consisting of an isocratic pump, a 2414 refractive index detector, a 2996 photodiode array detector, and a Waters Styragel HT4 column. A $N, N$-dimethylformamide solution containing $5 \mathrm{mM}$ of $\mathrm{NH}_{4} \mathrm{PF}_{6}$ was used as eluent at a flow rate of $0.5 \mathrm{~mL} \mathrm{~min}^{-1}$ and a column temperature of $50{ }^{\circ} \mathrm{C}$.
Molar masses were calculated against poly(methyl methacrylate) standards.

Proton nuclear magnetic resonance $\left({ }^{1} \mathrm{H}\right.$ NMR) spectra were recorded on a Varian Gemini $400 \mathrm{MHz}$ spectrometer at room temperature using deuterated chloroform $\left(\mathrm{CDCl}_{3}\right)$.

\section{Synthesis and characteristics of the diblock copolymer}

The PEtOx $\mathrm{x}_{67}-b-\mathrm{NonOx}_{33}$ diblock copolymer was synthesized by sequential cationic ring opening polymerizations under microwave irradiation; a detailed description of this synthetic method can be found elsewhere. ${ }^{34}$ The diblock copolymers showed narrow monomodal molar mass distributions as revealed by sizeexclusion chromatography (SEC). ${ }^{1} \mathrm{H}$ NMR spectroscopy in combination with SEC measurements was utilized for the determination of the composition of the different block copolymers. Thus, the investigated diblock copolymer $\mathrm{PEtOx}_{67}-b$ $\mathrm{NonOx}_{33}$ had a $M_{n}$ value of $9.4 \mathrm{kDa}(\mathrm{PDI}=1.35)$ as revealed by SEC analysis $\left(M_{n}\right.$ theor. $\left.=12.6 \mathrm{kDa}\right)$. The block compositions obtained by ${ }^{1} \mathrm{H}$ NMR spectroscopy for this material were DP = 67 and $\mathrm{DP}=33$ for the poly(2-ethyl-2-oxazoline) (PEtOx) and poly(2-nonyl-2-oxazoline) (PNonOx) blocks, respectively.

\section{Self-assembly and characterization of the block copolymer nanocarriers}

The self-assembled nanocarriers formed by the amphiphilic diblock copolymer $\mathrm{PEtOx}_{67}-b$-NonOx 33 utilized in this work were prepared utilizing the co-solvent method as reported elsewhere. ${ }^{10,11,15-17,35}$ The bulk diblock copolymer PEtOx $_{67}-b$ $\mathrm{NonOx}_{33}$ was dissolved in a thermodynamically good solvent for the two blocks (in acetone at $50{ }^{\circ} \mathrm{C}$ ) at a concentration of 10

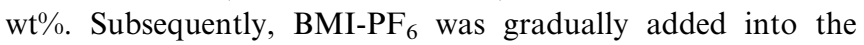
polymeric solution as a selective precipitant for the block composed of PNonOx under vigorous stirring in order to reach a concentration of $0.5 \mathrm{wt} \%$ of the polymeric material. The removal of the co-solvent from the nanocarrier dispersion was achieved by placing the vials in a vacuum oven at $40{ }^{\circ} \mathrm{C}$ for at least 24 hours. DLS measurements revealed that the investigated self-assembled nanocarriers in $\mathrm{BMI}^{-\mathrm{PF}_{6}}$ had a hydrodynamic radius $\left(R_{\mathrm{h}}\right)$ of $118 \mathrm{~nm}$ and a polydispersity index (PDI) of 0.29. These dimensions exceed the estimated sizes that would have been expected if micellar systems are formed, even if a complete stretching of the macromolecules is assumed. Thus, it is suggested that the structure of these self-assemblies is more complex and the term 'nanocarrier' is introduced to refer to these nanostructures.

\section{Dye loading of self-assembled nanocarriers}

The encapsulation of three different organic dyes in the cores of the self-assembled nanocarriers formed by the $\mathrm{PEtOx}_{67}-b$ $\mathrm{NonOx}_{33}$ diblock copolymer in the presence of the hydrophobic IL BMI-PF 6 was achieved by placing predetermined amounts of the corresponding dye and the diblock copolymer (10 $\mathrm{wt} \%$ of the dye with respect to the total amount of block copolymer) in a vial before the addition of the non-selective solvent (acetone in this case); the rest of the encapsulation procedure was identical to the preparation method explained above for the self-assembled diblock copolymer nanocarriers. The investigated organic dyes 
(Scheme 1) were disperse red 1 ( $N$-ethyl- $N$-(2-hydroxyethyl)-4-(4nitrophenyl-azo)-aniline) (D1), disperse orange 3 (4-(4-nitrophenyl-azo)aniline) (D2), and methyl blue ( $\mathrm{pH}$ indicator) (D3). Both D1 and D2 show a poor solubility in water but they were found to be well-soluble in BMI-PF 6 . D3 is well-soluble in water but insoluble in $\mathrm{BMI}_{-} \mathrm{PF}_{6}$. In addition, $\mathrm{D} 3$ acts as a $\mathrm{pH}$ indicator which turns colorless in the $\mathrm{pH}$ range from 9.4 to 14 .

\section{Results and discussion}

Before investigating the dye loading and transfer, the formation of self-assembled nanostructures of $\mathrm{PEtOx}_{67}-b$ - $\mathrm{PNonOx}_{33}$ was investigated in $\mathrm{BMI}_{-} \mathrm{PF}_{6}$ by DLS revealing a hydrodynamic size of $118 \mathrm{~nm}$, which is significantly larger than the value previously reported in water for the same diblock copolymer, namely $36 \mathrm{~nm} .{ }^{34}$ Even though $118 \mathrm{~nm}$ is rather large for individual micelles, TEM on the nanocarriers in a similar IL, namely BMI-CF $\mathrm{SO}_{3}$, revealed the presence of individual spherical nanostructures with a size around $100 \mathrm{~nm}$ indicating that the coronas of these nanostructures might be swollen by the IL (see Fig. 1 and ESI $\dagger$ ). Attempts to obtain cryogenic TEM micrographs of the formed nanostructures directly in $\mathrm{BMI}_{-} \mathrm{PF}_{6}$, using experimental techniques reported elsewhere, ${ }^{15,16}$ were not successful for the investigated system. This was mainly because a suitable sample thickness could not be obtained in several attempts due to the complexity of freezing an IL solution. ${ }^{15,16}$ For this reason, it was decided to determine the size of the nanostructures by TEM utilizing a related IL, BMI-CF $\mathrm{SO}_{3}$, which turned out to be easier (for details see the ESI $\dagger$ ). Additional DLS measurements of the self-assembly process of the investigated diblock copolymer performed in the presence of the hydrophilic IL BMI$\mathrm{CF}_{3} \mathrm{SO}_{3}$ revealed the formation of self-assembled spherical nanostructures with a $R_{\mathrm{h}}$ value of $84 \mathrm{~nm}$ and a PDI value of 0.54 . Even though it is reported that the nature of the IL can have an

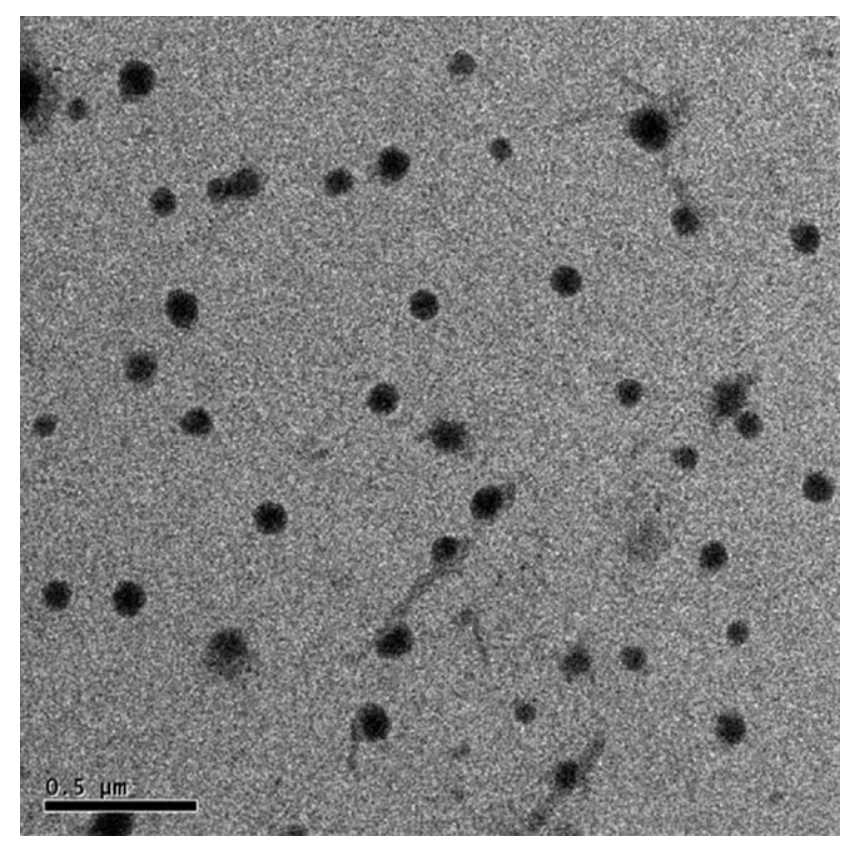

Fig. 1 TEM image of the $\mathrm{PEtOx}_{67}-b-\mathrm{NonOx}_{33}$ nanostructures in the BMI-CF $\mathrm{SO}_{3}$ IL. important influence on the self-assembly process of these nanostructures, ${ }^{11}$ this size from DLS is in a similar range as with BMI$\mathrm{PF}_{6}$ and, therefore, presumable individual spherical nanostructures will also be present in $\mathrm{BMI}_{-} \mathrm{PF}_{6}$. It was also reported that PNonOx is insoluble in water and in the mentioned ILs, and therefore the observed increased size of the nanocarriers in the ILs can be ascribed to stretching of the PEtOx chains in the corona and/or to the formation of micellar aggregates. ${ }^{11}$ It has also been reported that micellar structures formed of poly(butadiene)-block-poly(ethylene oxide) diblock copolymers can be thermoreversibly transferred between a $\mathrm{BMI}_{-} \mathrm{PF}_{6}$ IL phase and an aqueous phase where the micelle size and structure are preserved during the transfer process. ${ }^{10}$ As a consequence of the insolubility of $\mathrm{PNonOx}$ in $\mathrm{BMI}^{-\mathrm{PF}_{6}}$ and the possibility of thermoreversibly transferring this kind of structures between the phases of the investigated system, it may be anticipated that the IL is not transferred together with the nanocarriers into the aqueous phase.

Disperse red 1 dye (D1, Scheme 1) was subsequently loaded into the self-assembled $\mathrm{PEtOx}_{67}-b$ - $\mathrm{PNonOx}_{33}$ nanocarriers in $\mathrm{BMI}-\mathrm{PF}_{6}$ by simply preparing the nanocarriers in the presence of the dye. In fact, D1 is fully soluble in $\mathrm{BMI}^{-\mathrm{PF}_{6}}$ and, thus, it is expected that the dye is present in both the ILs and in the nanocarriers while D1 is hardly soluble in water, i.e. it is slightly soluble in the protonated form leading to a different absorption spectrum (Fig. 2). Fig. 2, top, shows that addition of a water

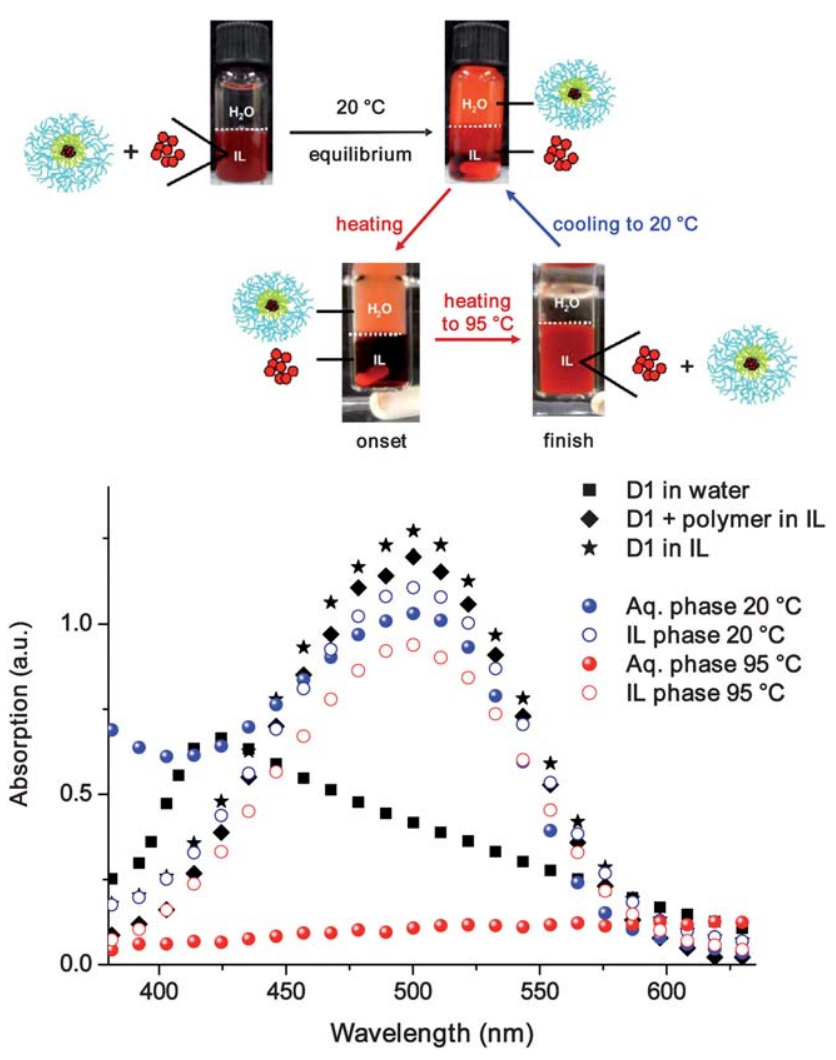

Fig. 2 Top: thermoreversible phase transfer organic dye molecules (disperse red 1 D1) encapsulated into self-assembled diblock copolymer

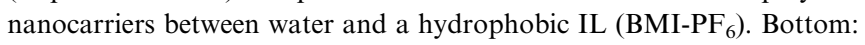
UV-Vis spectra of the aqueous and IL phases to investigate the location of the encapsulated D1 molecules at different temperatures. 
phase to the IL results in transfer of dye-loaded nanocarriers proving successful encapsulation and dye-transfer into the water phase..$^{11}$ The similar absorption of D1 in water in the presence of nanocarriers compared to the IL clearly indicated that the dye is inside the nanocarriers and it is not dissolved as the protonated form in water. Subsequent heating results in reversal of the nanocarrier shuttle from the water phase to the IL phase driven by the LCST transition of PEtOx. ${ }^{11}$ This reversible nanocarrier dye shuttle might be utilized for the delivery of precious reagents to the water phase as well as the possibility of reloading after all reagents would have been consumed. In addition, this nanocarrier shuttle might be used to switch the catalytic activity in the aqueous phase. The successful dye shuttling is confirmed by UVanalysis of the various phases (Fig. 2, bottom). Even though the scheme in Fig. 2 depicts an almost perfect nanocarrier dye shuttling procedure, it should be noted that this is an oversimplified representation. In fact, the partition coefficients of the nanocarriers between the phases, the dye in- and outside the nanocarriers as well as in between the phases will also play a minor role leading to a less perfect distribution of the components throughout the different phases.

In this initially discussed nanocarrier dye shuttle, D1 was present in a relatively large amount in the IL, which presumably is the driving force for loading the dye into the nanocarriers. To test this hypothesis, a similar experiment was performed with disperse orange 3 (D2, Scheme 1), which has a related structure to D1. Again the nanocarriers were prepared in the presence of a large amount of D2 and after addition of water, the nanocarriers loaded with D2 are transferred to the water phase demonstrating that the guest transfer is applicable to various guest molecules. To investigate the reversibility of the shuttling process, the IL still containing excess of $\mathrm{D} 2$ was replaced with the pure IL. Upon heating the nanocarriers comprising D2 are fully transferred to the IL leaving a colorless water phase (Fig. 3). Subsequent equilibration at ambient temperature resulted in the delivery of the D2 to the IL phase and transfer of practically empty nanocarriers into the water phase, which was evident from the visual absence of orange-yellowish colour in the final aqueous phase. As such, it is demonstrated that the nanocarrier dye shuttle can be used to deliver an IL soluble reagent to the IL and that the empty nanocarriers can be recovered in the water phase.

In a final experiment, the use of a water-soluble and BMI-PF 6 insoluble dye, methyl blue (D3, Scheme 1), was evaluated for micellar dye shuttling processes. A reference experiment of D3 in water on top of the IL phase resulted in vanishing of the blue color indicative of an increase in solution pH (Fig. 4a). At the initial stage the aqueous and $\mathrm{BMI}-\mathrm{PF}_{6}$ phases had apparent $\mathrm{pH}$

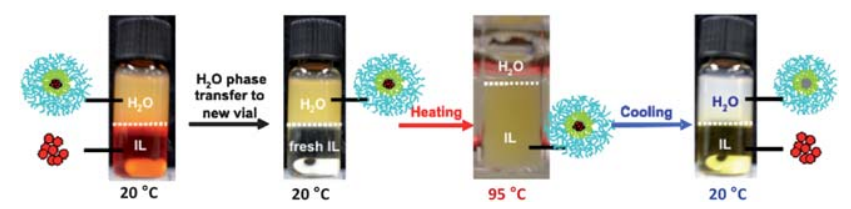

Fig. 3 Graphical overview of the thermoreversible phase transfer of D2 (disperse orange 3) encapsulated into the cores of self-assembled diblock copolymer nanocarriers (biphasic system: water and $\mathrm{BMI}_{-} \mathrm{PF}_{6}$ ) comprising transfer of the loaded micelles to the water phase followed by the delivery of the encapsulated D2 into a new hydrophobic IL phase and recovery of the utilized nanocarriers in the water phase. values of 7.5 and 5.8, respectively. After vanishing of the blue color, the aqueous and $\mathrm{BMI}_{-} \mathrm{PF}_{6}$ phases revealed apparent $\mathrm{pH}$ values of 11.9 and 8.8 , respectively. It was found that this large increase in $\mathrm{pH}$ value in the aqueous phase is mainly due to the presence of traces of impurity (i.e., 1-methylimidazole as precursor material of the synthesis of $\mathrm{BMI}^{-\mathrm{PF}_{6}}$ ) in the IL phase. Note that the IL utilized in this investigation was of synthesis grade. In fact, a separate experiment conducted with high purity $\mathrm{BMI}-\mathrm{PF}_{6}$ did not yield the observed $\mathrm{pH}$ changes in the described

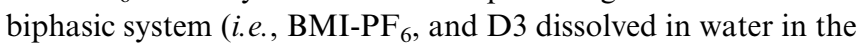
absence of discussed polymeric nanocarriers). However, upon adding a small amount of 1-methylimidazole to the latter system, the described $\mathrm{pH}$ changes arose as described above. 1-Methylimidazole has basic properties and provokes that the $\mathrm{pH}$ of the investigated system shifts to higher values. The presence of traces of 1-methylimidazolium was found to conveniently illustrate the protective properties of the investigated nanocarriers as a confined environment for $\mathrm{pH}$-sensitive guest moieties is discussed next. It is worth mentioning that the cases of phase transfer dye molecules corresponding to D1 and D2-addressed above - are unlikely to be affected by the presence of traces of 1methylimidazolium and its related $\mathrm{pH}$ change due to the fact that the solubility of these dyes is not affected by the observed $\mathrm{pH}$ changes. In addition, the utilized non-ionic polyamide is also $\mathrm{pH}$ insensitive.

When the block copolymer nanocarriers were present in the biphasic water-IL system, the color of the dye was retained even after repetitive shuttling as shown in Fig. 4. This clearly

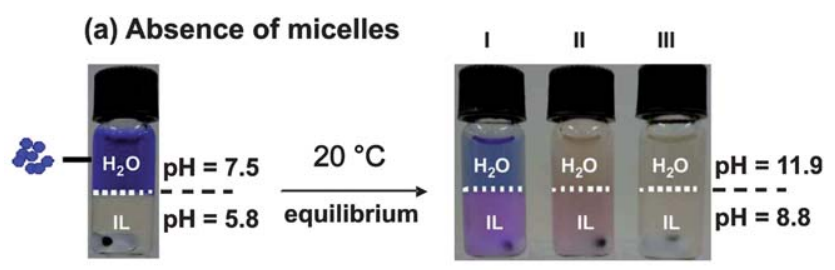

(b) Presence of micelles
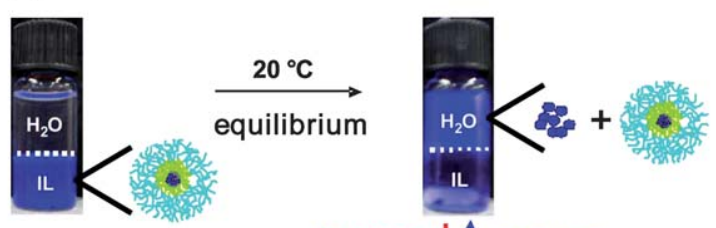

Initial system (1)

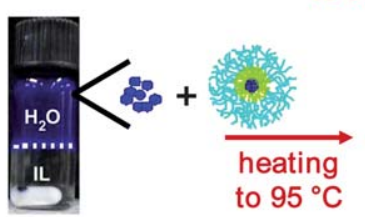

heating $\mid \hat{\mid}$ cooling to $95^{\circ} \mathrm{C}$ to $20^{\circ} \mathrm{C}$

Initial system (2)

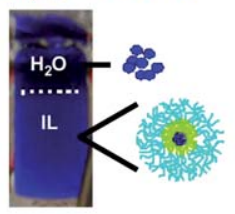

Fig. 4 (a) Decoloration of D3 (methyl blue) in a water-IL biphasic system after equilibrating at $20^{\circ} \mathrm{C}$ for $0.5 \mathrm{~h}$ (I), $2 \mathrm{~h}$ (II) and $24 \mathrm{~h}$ (III). (b) In the presence of the $\mathrm{PEtOx}_{67}-b$-PNonOx $\mathrm{P}_{33}$ self-assembled nanocarriers the changes of $\mathrm{pH}$ in the biphasic system do not affect the color of D3 molecules indicating that the carriers act as confined protective environments while still allowing nanocarrier dye shuttling. 
demonstrates that the encapsulation of the methyl blue dye into the nanocarriers shields the dye from the environment and, thereby, protects it from losing its color due to the $\mathrm{pH}$ changes observed in the reference system (Fig. 4a), at least within the time frame of our experiments $(24 \mathrm{~h})$. The initial dye loaded nanocarriers were prepared in either water or the IL, whereby the initial water phase contains both encapsulated and free methyl blue dye molecules while the IL phase only comprises dye-loaded nanocarriers due to insolubility of the dye in the IL. After addition of the second phase and a first equilibration at ambient temperature (for dye in the water phase) or elevated temperature (for dye in the IL phase), the initial history of the sample preparation is erased (at equilibrium at room temperature a slight bluish color remains in the IL phase, most likely a result of partitioning driven by the rather high dye concentration in the experiment) and both routes lead to a similar nanocarrier dye shuttle in between the two phases: at low temperatures the majority of the dye-loaded nanocarriers and free dye are present in the aqueous phase while at elevated temperatures the nanocarriers with incorporated dyes are present in the IL and the excess dye remains in the aqueous phase.

\section{Conclusions}

Based on the observations discussed above, three main conclusions can be drawn for the investigated nanocarrier dye shuttling systems. (1) Thermoreversible phase transfer of encapsulated dye molecules can occur when the amount of dye in the system exceeds considerably the amount of dye that can be encapsulated into the core of self-assembled nanocarriers. (2) One direction phase transfer and delivery of previously encapsulated hydrophobic dye molecules can occur from water to the IL when the IL phase is originally free of dye molecules. The reversed delivery can be achieved using a water-soluble dye. (3) The recovery of previously utilized nanocarriers and their reloading with new dye molecules are feasible.

These conclusions clearly indicate that the presence and concentration of excess of molecules, that can be encapsulated, in one of the phases represent important factors to achieve either its reversible transfer (case of catalytically active species) or its delivery into the other phase (shuttling of small amounts into the second phase for, e.g., chemical conversions).

All together, the nanocarrier dye shuttling approach presented in this work is envisioned to facilitate the design and development of advanced heterogeneous nanocarrier catalytic systems with interesting features such as the recovery of expensive or toxic catalysts from the final products, more efficient separation processes, the delivery of highly accurate amounts of chemical substances in multiphase systems, or the recovery of expensive self-assembled nanocarriers for further use.

\section{Acknowledgements}

This research forms part of the research program of the Dutch Polymer Institute, project \#401. The authors would like to thank the Fonds der Chemischen Industrie for financial support and the collaboration of Solvent Innovation $\mathrm{GmbH}$ in this work. This research has been carried out with the support of the Soft Matter
CryoTEM Research Unit, Department of Chemical Engineering and Chemistry, Eindhoven University of Technology.

\section{Notes and references}

1 M. Deetlefs and K. R. Seddon, Chim. Oggi, 2006, 24, 16-23.

2 P. Wassercheid and T. Welton, Ionic Liquids in Synthesis, Wiley-VCH Verlag GmbH, Weinheim, 2003.

3 C. Guerrero-Sanchez, T. Lara-Ceniceros, E. Jimenez-Regalado, M. Rasa and U. S. Schubert, Adv. Mater., 2007, 19, 1740-1747.

4 C. Guerrero-Sanchez, T. Erdmenger, P. Sereda, D. Wouters and U. S. Schubert, Chem.-Eur. J., 2006, 12, 9036-9045.

5 R. P. Swatloski, S. K. Spear, J. D. Holbrey and R. D. Rogers, J. Am. Chem. Soc., 2002, 124, 4974-4975.

6 P. Kubisa, J. Polym. Sci., Part A: Polym. Chem., 2005, 43, 4675-4683.

7 A. R. Choudhury, N. Winterton, A. Steiner, A. I. Cooper and K. A. Johnson, J. Am. Chem. Soc., 2005, 127, 16792-16793.

8 C. Guerrero-Sanchez, R. Hoogenboom and U. S. Schubert, Chem. Commun., 2006, 3797-3799.

9 C. Guerrero-Sanchez, M. Lobert, R. Hoogenboom and U. S. Schubert, Macromol. Rapid Commun., 2007, 28, 456-464.

10 Y. He and T. P. Lodge, J. Am. Chem. Soc., 2006, 128, 12666-12667.

11 C. Guerrero-Sanchez, J. F. Gohy, C. D'Haese, H. M. L. Thijs, R. Hoogenboom and U. S. Schubert, Chem. Commun., 2008, 27532755.

12 Z. Bai, Y. He and T. P. Lodge, Langmuir, 2008, 24, 5284-5290.

13 Z. Bai, Y. He, N. P. Young and T. P. Lodge, Macromolecules, 2008, 41, 6615-6617.

14 T. Ueki and M. Watanabe, Macromolecules, 2008, 41, 3739-3749.

15 Y. He, Z. Li, P. Simone and T. P. Lodge, J. Am. Chem. Soc., 2006, 128, 2745-2750.

16 P. M. Simone and T. P. Lodge, Macromol. Chem. Phys., 2007, 208, 339-348.

17 C. Guerrero-Sanchez, J. F. Gohy, D. Wouters, S. Hoeppener, H. M. L. Thijs, C. Ott, R. Hoogenboom and U. S. Schubert, PMSE Prepr., 2007, 96, 936-937.

18 Y. He and T. P. Lodge, Macromolecules, 2008, 41, 167-174.

19 Y. He, P. G. Boswell, P. Bühlmann and T. P. Lodge, J. Phys. Chem. B, 2007, 111, 4645-4652.

20 J. H. Cho, J. Lee, Y. He, B. S. Kim, T. P. Lodge and C. D. Frisbie, Adv. Mater., 2008, 20, 686-690.

21 P. M. Simone and T. P. Lodge, Macromolecules, 2008, 41, 1753-1759.

22 V. Chechik, M. Zhao and R. M. Crooks, J. Am. Chem. Soc., 1999, 121, 4910-4911.

23 T. P. Lodge, A. Rasdal, Z. Li and M. A. Hillmyer, J. Am. Chem. Soc., 2005, 127, 17608-17609.

24 M. A. R. Meier, J. F. Gohy, C. A. Fustin and U. S. Schubert, J. Am. Chem. Soc., 2004, 126, 11517-11521.

25 M. Kramer, J. F. Stumbe, H. Turk, S. Krause, A. Komp, L. Delineau, S. Prokhorova, H. Kautz and R. Haag, Angew. Chem., Int. Ed., 2002, 41, 4252-4256.

26 M. E. Piotti, F. Rivera, R. Bond, C. J. Hawker and J. M. J. Frechet, J. Am. Chem. Soc., 1999, 121, 9471-9472.

27 M. Filali, M. A. R. Meier, U. S. Schubert and J. F. Gohy, Langmuir, 2005, 21, 7995-8000.

28 O. G. Schramm, G. M. Pavlov, M. A. R. Meier, R. Hoogenboom and U. S. Schubert, Macromolecules, 2009, 42, 1808-1816.

29 C. Allen, J. Han, Y. Yu, D. Maysinger and A. Eisenberg, J. Controlled Release, 2000, 63, 275-286.

30 O. G. Schramm, M. A. R. Meier, R. Hoogenboom, H. P. van Erp, J. F. Gohy and U. S. Schubert, Soft Matter, 2009, 5, 1662-1667.

31 T. Dwars, E. Paetzold and G. Oehme, Angew. Chem., Int. Ed., 2005, 44, 7174-7199.

32 Z. Bai and T. P. Lodge, J. Phys. Chem. B, 2009, 113, 14151-14157.

33 Z. Bai and T. P. Lodge, Langmuir, 2010, 26, 8887-8892.

34 R. Hoogenboom, F. Wiesbrock, H. Huang, M. A. M. Leenen, H. M. L. Thijs, S. F. G. M. van Nispen, M. van der Loop, C. A. Fustin, A. M. Jonas, J. F. Gohy and U. S. Schubert, Macromolecules, 2006, 39, 4719-4725.

35 C. Guerrero-Sanchez, D. Wouters, C. A. Fustin, J. F. Gohy, B. G. G. Lohmeijer and U. S. Schubert, Macromolecules, 2005, 38, 10185-10191. 